\title{
PHKA2 mutation spectrum in Korean patients with glycogen storage disease type IX: prevalence of deletion mutations
}

\author{
Rihwa Choi ${ }^{1}$, Hyung-Doo Park ${ }^{1 *}$, Ben Kang ${ }^{2}$, So Yoon Choi ${ }^{2}$, Chang-Seok Ki', Soo-Youn Lee ${ }^{1}$, Jong-Won Kim', \\ Junghan Song ${ }^{3}$ and Yon Ho Choe ${ }^{2^{*}}$
}

\begin{abstract}
Background: Molecular diagnosis of glycogen storage diseases (GSDs) is important to enable accurate diagnoses and make appropriate therapeutic plans. The aim of this study was to evaluate the PHKA2 mutation spectrum in Korean patients with GSD type IX.

Methods: Thirteen Korean patients were tested for PHKA2 mutations using direct sequencing and a multiplex polymerase chain reaction method. A comprehensive review of the literature on previously reported PHKA2 mutations in other ethnic populations was conducted for comparison.

Results: Among 13 patients tested, six unrelated male patients with GSD IX aged 2 to 6 years at the first diagnostic work-up for hepatomegaly with elevated aspartate transaminase (AST) and alanine transaminase (ALT) were found to have PHKA2 mutations. These patients had different PHKA2 mutations: five were known mutations (c.537 + 5G > A, c.884G > A [p.Arg295His], c.3210_3212delGAG [p.Arg1072del], exon 8 deletion, and exons 27-33 deletion) and one was a novel mutation (exons 18-33 deletion). Notably, the most common type of mutation was gross deletion, in contrast to other ethnic populations in which the most common mutation type was sequence variant.

Conclusions: This study expands our knowledge of the PHKA2 mutation spectrum of GSD IX. Considering the PHKA2 mutation spectrum in Korean patients with GSD IX, molecular diagnostic methods for deletions should be conducted in conjunction with direct sequence analysis to enable accurate molecular diagnosis of this disease in the Korean population.
\end{abstract}

Keywords: Glycogen storage disease, Hepatomegaly, Inherited metabolic diseases, Korean, PHKA2

\section{Background}

Glycogen storage disease type IX (GSD IX) is caused by a general deficiency in phosphorylase kinase (PhK), which plays a major role in regulating the breakdown of glycogen. The PhK enzyme is composed of four copies each of four subunits $(\alpha, \beta, \gamma$, and $\delta$ [also known as calmodulin]), which are encoded by PHKA1 and PHKA2 for the $\alpha$ subunits, $P H K B$ for the $\beta$ subunits, PHKG1 and PHKG2 for the $\gamma$ subunits, and CALM1, CALM2,

\footnotetext{
* Correspondence: nayadoo@hanmail.net; yonho.choe@samsung.com 'Department of Laboratory Medicine and Genetics, Samsung Medical Center, Sungkyunkwan University School of Medicine, 81 Irwon-ro, Gangnam-gu, Seoul 135-710, Republic of Korea

${ }^{2}$ Department of Pediatrics, Samsung Medical Center, Sungkyunkwan

University School of Medicine, Seoul, Republic of Korea

Full list of author information is available at the end of the article
}

and CALM3 for the $\delta$ subunits [1]. There are autosomal recessive forms of GSD IX (caused by mutations in $P H K B$, PHKG1, PHKG2, CALM1, CALM2, and CALM3), a Xlinked liver form (caused by mutations in $P H K A 2$ ), and a $\mathrm{X}$-linked muscle form (caused by mutations in PHKA1). Specifically, the $\alpha$ subunits, which are encoded by the PHKA2 gene in liver and by PHKA1 in muscle, regulate the activity of the catalytic $\gamma$ subunits, which carry out the function of PhK. Mutations in PHKA2 are the most common cause of PhK deficiency [2].

The clinical manifestation of GSD IX stemming from PHKA2 mutation is characterized by childhood onset of hepatomegaly, growth retardation, fasting ketosis, and fasting hypoglycemia [3]. Symptoms and biochemical abnormalities in GSD IX patients have been reported to 
improve with age [4]. However, several GSD IX patients possessing PKHA2 mutations have been reported to progress to liver cirrhosis, or even present with cirrhosis at the time of diagnosis $[4,5]$.

GSD is a clinically and genetically heterogeneous group of diseases. Different types of GSD can sometimes be clinically indistinguishable; for example, both GSD VI and GSD IX often manifest with hepatomegaly with short stature [3]. Molecular diagnosis of GSD provides the advantage of avoiding invasive liver biopsy [6] and is an important starting point for appropriate therapeutic and monitoring plans [7]. Furthermore, molecular diagnosis allows determination of the inheritance pattern (autosomal recessive vs. X-linked) and DNA testing for other family members when the mutation in the proband is known.

The PHKA2 gene is located at chromosomal locus Xp22.2-p22.1 and consists of 33 exons spanning more than $65 \mathrm{~kb}$ and encoding a protein of 1,235 amino acids [8]. Sequence analysis of this X-linked gene can detect small intragenic deletions/insertions and missense, nonsense, and splice site mutations in males and females, and lack of amplification by PCR prior to sequence analysis can suggest a putative exonic or whole-gene deletion on the X chromosome in affected males [9]. Understanding the mutation spectrum of causative genes in different ethnic groups could provide background knowledge to develop a tailored diagnostic approach for different patient populations [7].

To date, data on PHKA2 mutations in Korean patients with GSD IX are available for only two cases. Thus, the aim of this study was to evaluate the mutation spectrum in Korean patients with GSD IX and compare it with previously reported mutation spectra in other ethnic populations.

\section{Methods}

\section{Study population}

From May 2010 to April 2015, blood samples from 13 unrelated Korean children were collected for PHKA2 mutation analysis at Samsung Medical Center. The study population included two female patients with hepatomegaly and elevated aspartate transaminase (AST) and/ or alanine transaminase (ALT) who were undergoing PHKA2 sequencing because no pathogenic mutations in G6PC (for GSD Ia), which is known to a common causative gene of glycogen storage disease in the Korean population, were identified during diagnostic work up for their hepatomegaly $[10,11]$. Blood samples from healthy individuals, who visited the health promotion center at Samsung Medical Center for regular health checkups without any clinical symptoms or signs of illnesses, and who volunteered for blood sampling, were also collected as negative controls for identified mutations. This study was conducted according to the guidelines laid down in the Declaration of Helsinki and all procedures involving human subjects were approved by the Institutional Review Board of Samsung Medical Center. Written informed consent was obtained from all subjects and/or their parents.

\section{PhK enzyme activity}

$\mathrm{PhK}$ enzyme activity in erythrocytes was measured according to a previously described method [12, 13]. The results of enzyme activity were expressed as $\mu \mathrm{mol} / \mathrm{mi}$ nute $(\mathrm{min}) / \mathrm{g}$ hemoglobin $(\mathrm{gHb})$. The reference range of phosphorylase b kinase enzyme activity in the laboratory was 100.0 to $250.0 \mu \mathrm{mol} / \mathrm{hr} / \mathrm{g} \mathrm{Hb}$ [12], which was validated using erythrocytes from healthy subjects. Each test was performed simultaneously in duplicate and with erythrocytes obtained from two different healthy subjects. Test results were accepted when the results of healthy control samples were within the reference range and the coefficient of variation of duplicated results was less than $10 \%$.

\section{Quantitation of liver glycogen}

Liver glycogen was measured according to a previously described method [14]. Fresh liver samples, $45 \mu \mathrm{L}$ in volume, were homogenized, heated at $95{ }^{\circ} \mathrm{C}$ for $5 \mathrm{~min}$, and mixed with $45 \mu \mathrm{L}$ of $0.1 \mathrm{M}$ sodium acetate buffer ( $\mathrm{pH} 4.8$ ), and $9 \mu \mathrm{L}$ of amyloglucosidase. Each homogenate was sonicated and incubated at $37^{\circ} \mathrm{C}$ for $30 \mathrm{~min}$ and then at $95{ }^{\circ} \mathrm{C}$ for $5 \mathrm{~min}$. Glycogen levels per gram of wet liver were calculated as glucose released by amyloglucosidase; i.e., glucose level after incubation with amyloglucosidase minus that without enzyme. The results were expressed as \% glycogen/g wet liver weight. The reference range of liver glycogen in the laboratory was 1.0 to $6.0 \%$ per gram of wet liver weight.

\section{PHKA2 mutation analysis}

Human genomic DNA was prepared from frozen white blood cells using a Wizard genomic DNA purification kit (Promega, Madison, WI, USA) according to the manufacturer's recommendations. All 33 exons and the flanking regions of the PHKA2 gene were amplified by polymerase chain reaction $(\mathrm{PCR})$ using primers designed by the authors (Table 1) with a thermal cycler (Model 970; Applied Biosystems, Foster City, CA, USA). Direct sequencing of the DNA was performed using the ABI Prism 3100 Genetic Analyzer (Applied Biosystems) with the BigDye Terminator Cycle Sequencing-Ready Reaction Kit (Applied Biosystems). Nucleotides are numbered from the first adenine of the ATG translation initiation codon in the PHKA2 cDNA Reference Sequence NM_000292.2.

To detect single or multiple exon deletions, a multiplex PCR method was performed using primers designed by the 
Table 1 Primers used for PCR, sequencing, and multiplex PCR for PHKA2 mutation analysis

\begin{tabular}{|c|c|c|c|}
\hline Exon & Forward primer sequence & Reverse primer sequence & Product size (bp) \\
\hline \multicolumn{4}{|c|}{ PHKA2 } \\
\hline 1 & CCATCCCAAGAACCGACTAA & GCAACAGTTAGGTCCCCTGA & 395 \\
\hline 2 & AGGTCCCGGTCCTCATCTAC & GAGAGGCCTACACCCAAACA & 367 \\
\hline 3 & AGCCACAGTGATCAGGAGGT & AATGACATGGAATGCCCACT & 174 \\
\hline 4 & GCTGGGACATITAGGCAAG & CACATGGCCTGACACACTG & 467 \\
\hline 5 & СCTTCCCTCTITTCGGAGAT & GCAGTTTGTGTGTGGAGGTG & 419 \\
\hline 6 & GGCTGCAGGAACATAAAGGA & CCAGGACGGAGCACTCTTAC & 401 \\
\hline 7 & TTGCTTAATGAAAAAGGAACACC & CTAGCTTGTGAGGCCAGAGG & 349 \\
\hline 8 & TGACTTCTCGCCTGAGGAAT & ACCTCATGGGGAACTGAGG & 427 \\
\hline 9 & TATCTGCCTTGGTGGCTITT & CCAGCTCACCGTCCCTACTA & 434 \\
\hline 10 & TCAGTCAAGCATGGGAAACC & CTCTGCCCAAATTGCAGAAT & 414 \\
\hline 11 & CCGATCGTGTITAGCTCCTC & TCCCAAAGTGCTGGGATTAC & 475 \\
\hline 12 & ATTGGCCTGGAGGATGAGTA & TGGACACACAAGGCTGAGAG & 399 \\
\hline 13 & TGAATATGTTGAGCCCCAAA & CCCAGTTGCAATCAAGGTT & 428 \\
\hline 14 & ATGTCACCAGGCAGAAGAGG & CCGCCTGCTTTAGTTITTGT & 353 \\
\hline 15 & GAAGAACCAAGCCCCAAAAT & ACGCCTGTCTCAAAAAGCAT & 421 \\
\hline 16 & ACTGGGTGGATTGAAACGAG & AGAAGCCCCTTCAGTGCTTA & 389 \\
\hline 17 & CGGGAATCTTCTATGCCAGA & TGGTTCACCTCCCTATGTCC & 444 \\
\hline 18 & CCACATGGTTGTGCAAAAGT & CGGTTITAAACGGGCATT & 441 \\
\hline 19 & GCTTGCTACCCATGGTCACT & GGGGCATTTTGTTGTCTTCT & 364 \\
\hline 20 & GAGGCAAAGGTTGCAGTGAG & TGCAAGTCAGATTCCAGACAA & 412 \\
\hline 21 & GAAAACTGGAGCACAGCACA & CCATGTCAGGATGCAATGAG & 434 \\
\hline 22 & ACCACGTCCTGATGTTAGGC & ATGGGGCTCCTTCACAAGTA & 449 \\
\hline 23 & TCCCTGTCTGGGTTGCTTAG & AGACGCATCCATGTGACAGA & 382 \\
\hline 24 & TCTGTCACATGGATGCGTCT & TCTCCTGAGGCAGACACACA & 303 \\
\hline 25 & ACAGCCTTCCTCAGAGTGGA & GGATGCTGGGTTCGAGATAA & 321 \\
\hline 26 & TTCAGCCCCAAAGCAATAG & ACACTGCGAGCAAGTCTCAA & 435 \\
\hline 27 & CAGAGAAGGCCCTCATTGTC & GGACAGGGGTGTGTTCAGAT & 376 \\
\hline 28 & CCATGAGAAATGCACTCGAA & ATAGAGCCGCCCTCTACACA & 337 \\
\hline 29 & СTCTGCTGCTGCTITCTGTG & GACGGAGAACAAAGCTCAGG & 365 \\
\hline 30 & GTGGTGTTCTGGCATTTGTG & ATCCTCAGGGCTGTGTGTTT & 383 \\
\hline 31 & TGTTCCATCGAAAACACAGC & TGATGCCAATAAATGCTGGA & 436 \\
\hline 32 & GCTACGGTCACCCTTGGTTA & TITICCCCATCATCTGTGA & 410 \\
\hline 33 & CTCAGAAGGCCAAGGCTCTA & CTGATGGGACATGCTTTCCT & 415 \\
\hline \multicolumn{4}{|c|}{$M E C P 2^{a}$} \\
\hline 4 & CGCTCTGCCCTATCTCTGAC & TCCCCTCGGTGTTTGTACTT & 1061 \\
\hline
\end{tabular}

${ }^{\mathrm{a}}$ Used as amplification control of MECP2 gene exon 4 located in Xq28 for multiplex PCR

authors (Table 1). All tests were performed concurrently on negative control samples from healthy individuals.

Additionally, a comprehensive review of the literature on previously reported PHKA2 mutations in Asian populations was conducted. The Leiden Open Variation Database (LOVD, http://www.LOVD.nl/PHKA2) and Human Gene Mutation Database (HGMD, h https://portal.biobase-international.com/cgibin/portal/login.cgi were checked for previously reported sequence variants. Variants identified in this study were checked through public databases. Common and rare variants present in the PHKA2 gene in Korean population could be obtained from the Korean Reference Genome Database (http://152.99.75.168/KRGDB/) and compared with other ethnic populations through the Exome Aggregation Consortium (ExAC), which aggregates over 60,000 
human exomes. The 1000 Genomes Project database (http://browser.1000genomes.org), the National Heart, Lung, and Blood Institute (NHLBI) Exome Sequencing Project (ESP) database (http://evs.gs.washington.edu/ EVS), and the NCBI database of Single Nucleotide Polymorphisms (dbSNPs) were also checked for previously reported sequence variants.

The pathogenicity of missense variants was evaluated by in silico analyses using Sorting Intolerant from Tolerant (SIFT) (http://sift.jcvi.org/), and Polymorphism Phenotyping v.2 (PolyPhen-2) (http://genetics.bwh.harvard.edu/ pph2/) prediction programs. Human Splice Finder software (http://www.umd.be/HSF3/) was used to predict splicing signals [15].

\section{Results}

During the study period, six patients were identified as having PHKA2 mutations. All six patients were male. Clinical and biological information at the time of diagnosis are detailed in Tables 2 and 3. Median age at first diagnostic work-up was 3 years (range 2-4 years). All patients presented with hepatomegaly and elevated AST and/or ALT as the first clinical features. Short stature, defined as height below the 3rd percentile for age by Korean Children and Adolescents Growth Standard [16], was observed in $33.3 \%$ (2/6 patients, case 3 and 4$)$ of GSD IX patients. The height of case 2, who had a missense mutation of c.884G > A (p.Arg295His), was within the 25th to 50th percentile. Among these six patients, only two had been tested for PhK enzyme activity in their erythrocytes, and both showed decreased PhK activity. Liver biopsies had been performed for five patients. Among them, the results of four (patients 3, 4, 5, and 6) were compatible with glycogen storage disease: Periodic acid-Schiff (PAS) staining was positive and PAS diastase stain was negative. One patient who was referred from an outside hospital was reported to be $\mathrm{PAS}^{+}$in liver biopsy, but limited information was available because the liver biopsy had been performed at the outside hospital. All patients initiated uncooked cornstarch therapy after diagnosis of GSD. None of the patients experienced symptomatic fasting hypoglycemia at the time of diagnosis or during the follow-up period. Glucose monitoring, in which blood glucose was measured upon waking in the morning in a fasting state, identified one episode of asymptomatic fasting hypoglycemia $(<70 \mathrm{mg} / \mathrm{dL})$ [17] in each of case 2 and case 6.

Each of the six patients had a different PHKA2 mutation. Among the six mutations, five were known mutations-c.537 + 5G > A [6], c.884G > A (p.Arg295His) [8], c.3210_3212delGAG (p.Arg1072del) [18, 19], exon 8 deletion [13], and exons 27-33 deletion (Fig. 1) [6] -and one was a novel mutation (exons 18-33 deletion). None of these mutations was observed in control population databases. The novel deletion mutation identified in case 5 was the largest deletion mutation (16 exons) ever reported among GSD IX patients, except for patients reported in the literature with full PHKA2 deletion who had no paternal X-chromosome (LOVD). However, there was a lack of formal information on whether these patients had Turner syndrome, and therefore only one copy of PHKA2. Caution is required when using information from public databases and it is necessary to clarify the status of these patients.

Our review of the literature on PHKA2 mutations in Asian populations with GSD IX is summarized in Table 4. Among 18 different PHKA2 mutations reported in Asian populations, nine (50\%) were splicing or deletion mutations. The overall PHKA2 mutation spectrum derived from a comprehensive literature review is summarized in Fig. 2. The most common PHKA2 mutation type among other ethnic groups and Asian populations

Table 2 Summary of the clinical features of six GSD IX patients with PHKA2 mutation

\begin{tabular}{|c|c|c|c|c|c|c|c|c|c|c|c|c|}
\hline $\begin{array}{l}\text { Patient } \\
\text { no. }\end{array}$ & Sex & Age $(y r)^{a}$ & $\begin{array}{l}\text { Hepato- } \\
\text { megaly }\end{array}$ & Epistaxis & $\begin{array}{l}\text { Short } \\
\text { stature }^{b}\end{array}$ & $\begin{array}{l}\text { Hypo- } \\
\text { glycemia }\end{array}$ & $\begin{array}{l}\text { Hyper- } \\
\text { uricemiac }^{c}\end{array}$ & $\begin{array}{l}\text { Hyper-lactic } \\
\text { acidemia }^{d}\end{array}$ & $\begin{array}{l}\text { Hyper- } \\
\text { lipidemia }\end{array}$ & $\begin{array}{l}\text { CK } \\
\text { increase }^{f}\end{array}$ & $\begin{array}{l}\text { Persistent } \\
\text { hepatomegaly }\end{array}$ & $\begin{array}{l}\text { Follow-up } \\
\text { period (mo) }\end{array}$ \\
\hline 1 & $M$ & 2 & Yes & No & No & No & No & Yes & Yes & No & Yes & 13 \\
\hline 2 & $M$ & 6 & Yes & No & No & $Y_{e s}^{g}$ & No & Yes & No & No & No & 26 \\
\hline 3 & $M$ & 4 & Yes & Yes & Yes & No & No & Yes & No & No & No & 178 \\
\hline $4^{h}$ & $M$ & 2 & Yes & Yes & Yes & No & No & Yes & Yes & No & $Y_{e s}^{i}$ & 48 \\
\hline 5 & $M$ & 2 & Yes & No & No & No & No & Yes & Yes & No & Yes & 33 \\
\hline 6 & $M$ & 4 & Yes & No & No & $Y_{e s}^{g}$ & No & Yes & No & Not done & Yes & 4 \\
\hline
\end{tabular}

${ }^{\mathrm{a} A g e}$ at first diagnostic workup



'Uric acid level $>7.2 \mathrm{mg} / \mathrm{dL}$ for at least one measurement during follow-up period

${ }^{\mathrm{d}}$ Lactic acid level $>2.2 \mathrm{mmol} / \mathrm{L}$ for at least one measurement during follow-up period

${ }^{\mathrm{e}}$ Total cholesterol $>240 \mathrm{mg} / \mathrm{dL}$ and/or triglyceride $>200 \mathrm{mg} / \mathrm{dL}$ for at least one measurement during follow-up period

f Serum creatine kinase $>204 \mathrm{IU} / \mathrm{L}$ for at least one measurement during follow-up period

${ }^{9}$ Glucose monitoring in which blood glucose was measured upon waking in the morning in a fasting state identified one episode of asymptomatic fasting

hypoglycemia $(<70 \mathrm{mg} / \mathrm{dL}$ ) during the follow-up period. The patient did not experience symptomatic hypoglycemia

${ }^{h}$ X-linked inheritance was identified by maternal PHKA2 mutation analysis

'Mild improvement from two fingerbreadths to one fingerbreadth during follow-up period 
Table 3 Mutations found in six Korean patients with GSD type IX

\begin{tabular}{|c|c|c|c|c|c|c|c|c|}
\hline Case no. & Sex & $\operatorname{Age}^{a}(y r s)$ & $\begin{array}{l}\text { PhK in erythrocytes } \\
(\mu \mathrm{mol} / \mathrm{min} / \mathrm{gHb})\end{array}$ & Liver glycogen ${ }^{c}$ & Liver biopsy & Identified mutation & Mutation type & Ref. \\
\hline 1 & $M$ & 2 & Not done & Not done & Not done & $c .537+5 G>A$ & Splicing & {$[6]$} \\
\hline 2 & M & 6 & Not done & Not done & $\operatorname{PAS}(+)^{d}$ & c.884G > A (p.Arg295His) & Missense & {$[8]$} \\
\hline 3 & M & 4 & Not done & $\begin{array}{l}8.8 \% \text { (initial), } \\
22.5 \%(f / u)\end{array}$ & $\begin{array}{l}\text { c/W GSD, PAS(+), } \\
\text { D-PAS(-) }\end{array}$ & $\begin{array}{l}\text { c.3210_3212delGAG } \\
\text { (p.Arg1072del) }\end{array}$ & Small deletion & {$[18,19]$} \\
\hline 4 & M & 2 & 6.57 & Not done & $c / w$ GSD & Exon 8 deletion & Gross deletion & [13] \\
\hline 5 & M & 2 & $\begin{array}{l}\text { Activity below } \\
\text { detection level }\end{array}$ & $25.1 \%$ & $\begin{array}{l}\text { C/W GSD, PAS(+), } \\
\text { D-PAS(-) }\end{array}$ & Exons $18-33$ deletion & Gross deletion & This study \\
\hline 6 & M & 4 & Not done & Not done & $\begin{array}{l}\text { c/w GSD, PAS(+), } \\
\text { D-PAS(-) }\end{array}$ & Exons 27-33 deletion & Gross deletion & {$[6]$} \\
\hline
\end{tabular}

$c / w$ compatible with, D-PAS Periodic acid-Schiff diastase stain, $f / u$ follow-up, PAS Periodic acid-Schiff stain, PhK Phosphorylase $b$ kinase, Ref references ${ }^{a}$ Age at first diagnostic workup due to clinical presentation (all patients presented with hepatomegaly with elevated aspartate aminotransferase and alanine aminotransferase)

${ }^{\mathrm{b}}$ Reference range of the laboratory was $100.0-250.0 \mu \mathrm{mol} / \mathrm{min} / \mathrm{gHb}$

${ }^{c}$ Reference range of the laboratory was $1-6 \% / g$ wet liver weight

${ }^{\mathrm{d} O n l y}$ limited information available on results of liver biopsy performed at outside hospital

other than Koreans was sequence variants, such as missense, nonsense, or frameshift mutations.

\section{Discussion}

In this study we examined the PHKA2 mutation spectrum in Korean patients with GSD IX. GSD IX is associated with a deficiency in liver PhK (caused by mutations in PHKA2, PHKB and PHKG2) or the muscle form of PhK (caused by mutations in PHKA1). Mutations in PHKA2 have been reported to be the most common cause of GSD IX (responsible for approximately $75 \%$ of cases) $[20,21]$. The second most common cause of liver PhK deficiency is mutations in PHKG2 (autosomal recessive), followed by $P H K B$ (also autosomal recessive) [3]. X-linked liver glycogenosis (XLG) caused by PHKA2 mutations can be divided into two subtypes: XLG I with no detectable activity of $\mathrm{phK}$ in liver and peripheral blood cells, and XLG II with normal activity in peripheral blood cells and deficiency in the liver [8]. Although several female GSD IX patients with PHKA2 mutations have been reported in other populations, including Western and other Asian populations [18, 22-24], only male patients were identified among our Korean patient population. The PHKA2 mutation spectrum is known to be distributed across the entirety of PHKA2 exons, with the exception of exon 13. Except for amino acids 420,
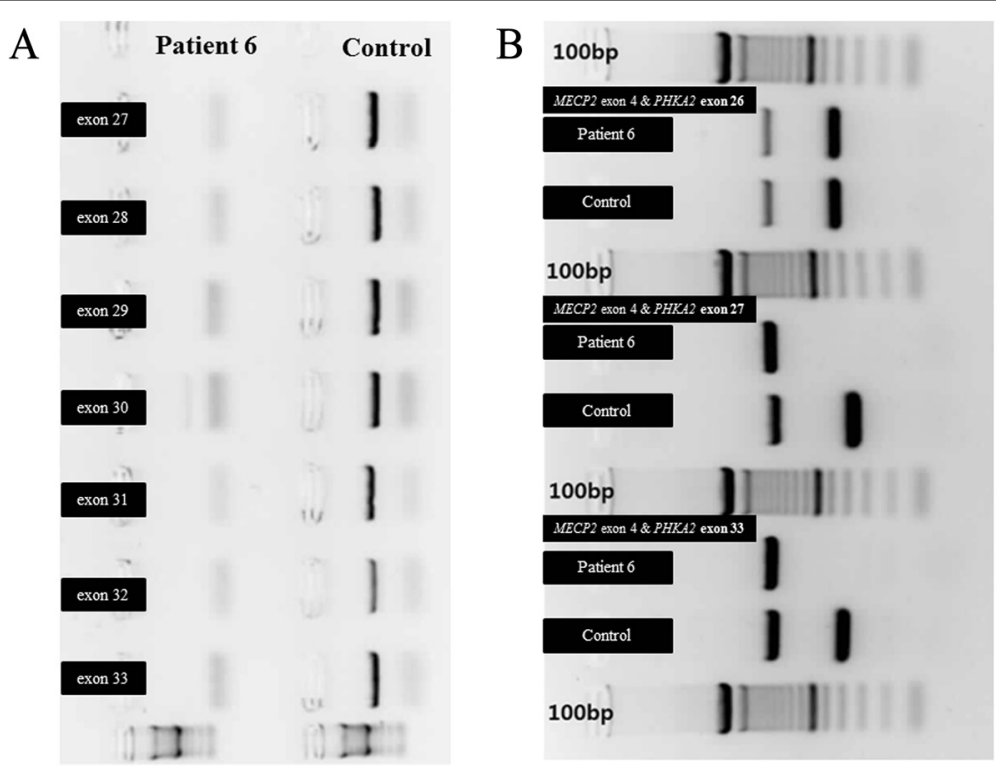

Fig. 1 Example of a large exonic deletion identified in patient 6. a Lack of amplification of PCR products from exon 27 to exon 30 of the PHKA2 gene in patient 6 compared with normal control. b Multiplex PCR results using control primers for amplification of MECP2 gene exon 4 (1,061 bp) located in Xq28 and test primers for amplification of exon 26 (435 bp), exon 27 (376 bp), and exon 33 (415 bp) 
Table 4 Reported PHKA2 mutations in Asian patients with glycogen storage disease (GSD) type IX

\begin{tabular}{|c|c|c|c|c|c|}
\hline Ethnicity & Exon number & Nucleotide change & Amino acid change & Mutation type & Reference \\
\hline \multicolumn{6}{|l|}{ Point mutation } \\
\hline Chinese & 2 & c.136delG & p.Asp46llefs*37 & Frameshift & {$[31]$} \\
\hline Japanese & 4 & c.346 T> G & p.Tyr116Asp & Missense & {$[32]$} \\
\hline Japanese & 6 & $c .578 \mathrm{G}>\mathrm{T}$ & p.Gly193Val & Missense & {$[32]$} \\
\hline Japanese & 9 & $\mathrm{c} .883 \mathrm{C}>\mathrm{T}$ & p.Arg295Cys & Missense & {$[25]$} \\
\hline Korean & 9 & $c .884 G>A^{a}$ & p.Arg295His & Missense & [8], This study \\
\hline Japanese & 15 & c. $1489 C>T$ & p.Arg 497* & Nonsense & {$[25]$} \\
\hline Japanese & 16 & c. $1697 \mathrm{~A}>\mathrm{T}$ & p.lle566Asn & Missense & [33] \\
\hline Japanese & 32 & c. $3505 C>T$ & p.Gln1169* & Nonsense & {$[25]$} \\
\hline Japanese, Chinese ${ }^{\mathrm{b}}$ & 33 & c. $3614 C>T$ & p.Pro1205Leu & Missense & {$[22,24,32,34]$} \\
\hline \multicolumn{6}{|l|}{ Splicing mutation } \\
\hline Japanese & 2 & c.79-1G > T & Exon 2 skipping & Splicing & {$[25]$} \\
\hline Korean & IVS5 & $c .537+5 G>A$ & $?^{c}$ & Splicing & [6], This study \\
\hline Japanese & 25 & c. $2675 A>G$ & Exon 25 skipping & Splicing & {$[32]$} \\
\hline Chinese & 30 & c. $3112-1 G>A$ & Exon 30 skipping & Splicing & {$[31]$} \\
\hline \multicolumn{6}{|l|}{ Deletion mutation } \\
\hline Korean & 30 & c.3210_3212delGAG & p.Arg1072del & Small deletion & {$[18,19]$, This study } \\
\hline Korean & 8 & & Exon 8 deletion & Gross deletion & [13], This study \\
\hline Korean & $18-33$ & & Exon 18-33 deletions & Gross deletion & This study \\
\hline Japanese & $20-26$ & & Exon $20-26$ deletions & Gross deletion & {$[29]$} \\
\hline Korean & $27-33$ & & Exon 27-33 deletions & Gross deletion & {$[6]$, This study } \\
\hline
\end{tabular}

423, and 432-434, the protein sequence of PHKA2 is strictly conserved among various mammals and zebrafish (Fig. 3). Exon 13, which corresponds to amino acids 416 to 442 , is in a six-hairpin glycosidase domain (amino acids 9 - 453) that contains pathogenic mutations including several missense mutations [2]. Although several missense variant alleles have been identified in exon 13 (ExAC), hemizygosity and lack of clinical information make interpretation of their clinical significance difficult. Further studies are needed to clarify the clinical significance of mutations in exon 13.

No recurrent mutations were observed among Korean GSD IX patients, although a missense PHKA2 mutation of p.Pro1205Leu suggestive of a founder mutation has been reported among Dutch patients [22]. This mutation has also been reported in Japanese and Chinese patients, but has not been observed in the Korean population [22, 24, 25].

In this study, we identified one novel and five known PHKA2 mutations. The missense mutation of c.884G > A
(p.Arg295His) has been reported in a patient with unusually severe clinical manifestation with marked ketosis and hyperlipidemia $[8,26]$ In our study, patient 2 carrying the same mutation showed a relatively mild clinical manifestation, with asymptomatic hepatomegaly and asymptomatic hypoglycemia and height and weight within the 25th to 50th percentiles, although he had experienced hyperlactic acidemia. This mutation has been reported previously in a patient with XLG I who had a pronounced PhK deficiency in both liver and erythrocytes (PhK activity was $10.0 \%$ of the control mean in both liver and erythrocytes) and in a patient with XLG II who had normal erythrocyte PhK activity (83.0\% of the control mean), therefore the significance of this mutation for biochemical function in specific tissues and clinical severity remains speculative [8]. This mutation was predicted to affect protein function by in silico analyses (SIFT and PolyPhen-2) and was predicted to alter splicing through activation of an exonic cryptic donor site by both Human Splice Finder software and by a machine-learning technique that scores 


\section{A PHKA2 Mutation type} (All reported mutations)

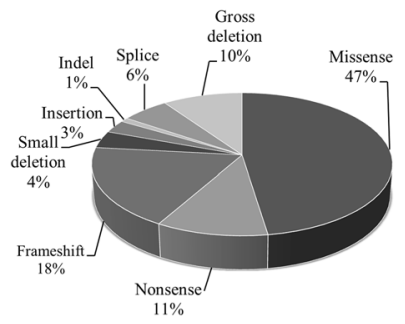

B PHKA2 Mutation type (Asian other than Korean)

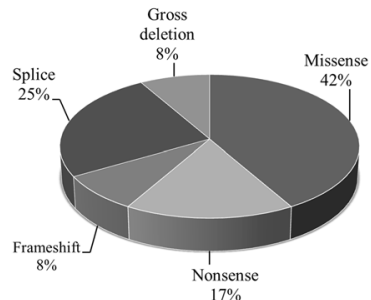

C PHKA2 Mutation type in Korean GSD IX patients

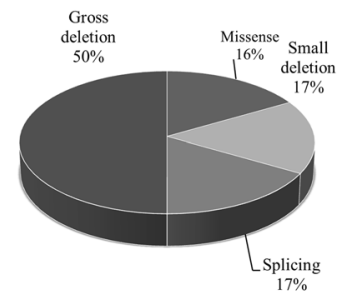

D PHKA2 mutation spectrum (All reported mutations)

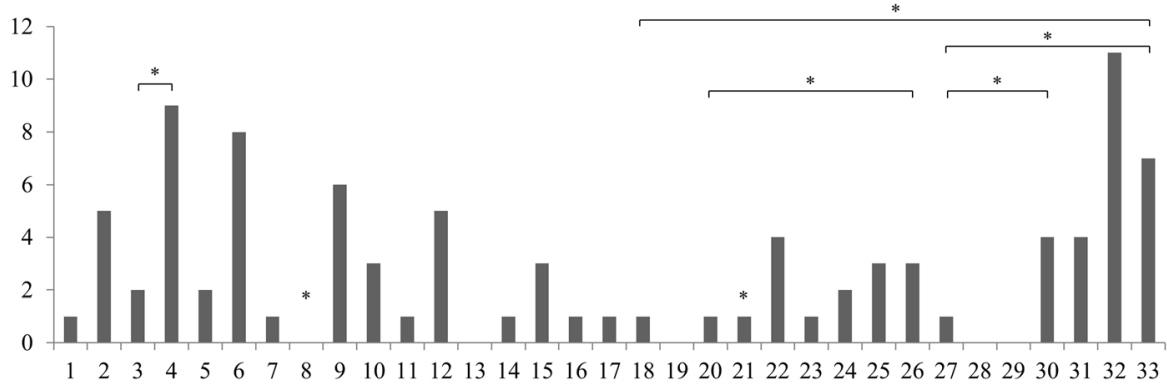

Fig. 2 Summary of reported PHKA2 mutation spectrum: a PHKA2 mutation types among all reported mutations in all ethnic populations; b PHKA2 mutation types identified in Asian GSD IX patients (other than Korean); c PHKA2 mutation types identified in Korean GSD IX patients; d Number of reported PHKA2 mutations in each exon (excluding gross deletions spanning more than one exon). The $x$-axis is the number of each coding exon and the $y$-axis is the number of identified mutations. ${ }^{*}$ Gross deletion spanning more than one exon designated by additional asterisk and lines

how strongly genetic variants affect RNA splicing [27]. The c.537 + 5G > A mutation has been reported in a male GSD IX patient of European ancestry who presented with hepatomegaly and growth delay that presented from 9 months of age [6]. This mutation was predicted to affect splicing through alteration of the wild-type donor site. Although in vitro analysis of the splicing effect of c.537+ $5 \mathrm{G}>\mathrm{A}$ was not performed, in vivo results confirmed phosphorylase $b$ kinase deficiency in the patient's lymphocytes (32 U/g, normal range 100-240 U/g) and erythrocytes $(0.7 \mathrm{U} / \mathrm{g} \mathrm{Hb}$, normal range 4-10 U/g Hb) [6]. Clinical manifestations of the patient carrying this mutation were reported to include growth delay, mild fasting hypoglycemia, post-prandial lactatemia, and elevated serum triglycerides, with age at onset of 9 months [6]. In our study, patient 1 carrying the same mutation had post-prandial lactatemia and elevated serum triglycerides in common, but no fasting hypoglycemia or growth delay. A small deletion mutation of c.3210_3212delGAG (p.Arg1072del) has been reported in a Finnish male GSD IX patient with hepatomegaly without short stature or hyperlactic acidemia [18] whose age at onset was 16 months. That patient was diagnosed with GSD IX by molecular diagnosis, but a PhK enzyme activity test was not performed [18]. In this study, the patient with the same mutation was of short stature and his liver glycogen concentrations increased over time $(8.8 \% / g$ wet liver weight at initial diagnosis and $22.5 \% / g$



Fig. 3 Evolutionary conservation of the amino acid residues for exon 13 on PHKA2 gene. Except for several amino acid sites, such as amino acid 420, 423, and 432-434, other amino acid residues are strictly conserved in various mammals and zebrafish 
wet liver weight at 2.5 year follow-up; reference range 1-6 \%/g wet liver weight). His age of onset was 4 years and he experienced hyperlactic academia, which was different from the Finnish male. A gross deletion mutation of exons 27-33 has been reported in a male GSD IX patient of European ancestry with PhK deficiency diagnosed by markedly decreased phosphorylase activities in his lymphocytes and erythrocytes, whose age at onset was 8 months [6]. Because PhK (defective PhK activities cause GSD IX) activates glycogen phosphorylase b (defective phosphorylase activities cause GSD VI) by phosphorylation, patients with GSD IX can have decreased phosphorylase activities [6]. Molecular studies and analysis of $\mathrm{PhK}$ are needed to make the correct diagnosis and avoid misdiagnosis of GSD VI [28]. The male patient in the previous literature was reported to have post-prandial lactatemia with elevated serum triglycerides [6]. In this study, patient 6 carrying the same large deletion had asymptomatic fasting hypoglycemia and post-prandial lactatemia but no elevated serum triglycerides. His age at first diagnostic workup was 4 years.

Of note, most mutations in Korean GSD IX patients were deletion or splicing mutations, except for one known missense mutation of c.884G > A (p.Arg295His) [8]. Fifty percent of Korean GSD IX patients had gross deletion mutations, and $83.3 \%$ had splicing or deletion mutations that were different from those reported in other ethnic populations. These results suggest that laboratory tests for large deletions in PHKA2 should be included in the variety of methods that may be used such as quantitative PCR, long-range PCR, multiplex ligation-dependent probe amplification, and that chromosomal microarrays including this gene/chromosome segment should be used as the first approach for Korean patients suspected to have GSD IX [9]. In this study, the presence of deletions in male GSD IX patients was initially implied by the lack of amplification of exons by PCR and additional multiplex PCR was then was employed to better define the deletion. Although only male patients had PHKA2 mutations in this study, multiplex PCR would be necessary to detect deletions in females with suspected GSD IX [9]. Methods to detect deletions would be important for patients of all ethnicities $[6,9,29]$.

Historically, diagnosis of GSD was based on enzymatic defects of each type of GSD or on liver histopathology compatible with GSD [6]. Recent molecular diagnostic approaches based on mutation analysis for diseasecausing genes associated with each type of GSD provide the advantages of avoiding invasive liver biopsy and allowing differentiation between several types of GSD with similar clinical findings [6].

Because of its variable clinical manifestations with mild symptoms, underestimation of GSD IX is possible.
For example, short stature was observed in $33.3 \%$ of patients in this study, which was comparable to previous studies in European and Argentinian populations [18] but different from a Canadian study that reported no patients with short stature [3]. Furthermore, the effect of PHKA2 mutations on tissues other than liver should be clarified through future studies. A recent study reported that genetic diagnosis for $\mathrm{X}$-linked mental retardation revealed several PHKA2 variants [30]. Although the natural history of GSD IX in Canadian patients has been reported, there are no reliable data on long-term outcomes for Korean GSD IX patients [3]. The study on 11 Canadian GSD IX patient with PHKA2 mutations with a follow-up period ranging from 1 to 16 years reported improved, normalized, or stable liver enzymes in all patients, and development of likely liver adenoma in one patient at the 5-year follow-up [3]. Detailed long-term natural history studies of Korean patients with GSD IX caused by PHKA2 mutation will be helpful to understand whether these patients are at increased risk of developing additional complications such as liver cirrhosis or hepatocellular carcinoma, or other conditions such as reproductive or mental disorders, later in life.

$\mathrm{PhK}$ deficiency is very complex and many genes play a key role in the GSD IX phenotype; the spectrum includes autosomal recessive forms of GSD IX (caused by mutations in PHKB, PHKG1, PHKG2, CALM1, CALM2, and $C A L M 3$ ) as well as the $\mathrm{X}$-linked liver form (caused by mutations in $P H K A 2$ ) and $\mathrm{X}$-linked muscle form (caused by mutations in PHKA1). Only PHKA2 mutations were studied in this study. Future studies involving comprehensive mutational analyses of multiple causative genes in the Korean population are needed.

\section{Conclusions}

In this study we summarized the PHKA2 mutation spectrum in Korean GSD IX patients and found that the most common mutation type was gross deletion. The present study expands our knowledge of the mutational spectrum in Korean GSD IX patients, which differs from that in other ethnic populations. Considering the PHKA2 mutation spectrum in Korean patients with GSD IX, molecular diagnostic methods for deletions should be combined with direct sequence analysis to provide accurate molecular diagnosis of this disease.

\footnotetext{
Competing interests

The authors declare that they have no competing interests.
}

\section{Authors' contributions}

RC acquisition of data, analyses and interpretation of data, preparation of the manuscript. HDP design, analyses and interpretation of data, revision of the manuscript. BK and SYC acquisition of clinical data. CSK and JWK molecular genetic studies and sequence alignment. SYL and JS biochemical assays and critical review of the manuscript. YHC design of the study, analysis of the clinical and laboratory data, and critical review of the manuscript. All authors read and approved the final manuscript. 


\section{Acknowledgments}

This study was supported by a grant from the Korea Health Technology R\&D Project, Ministry of Health \& Welfare, Republic of Korea (A120030).

\section{Author details}

'Department of Laboratory Medicine and Genetics, Samsung Medical Center, Sungkyunkwan University School of Medicine, 81 Irwon-ro, Gangnam-gu, Seoul 135-710, Republic of Korea. ${ }^{2}$ Department of Pediatrics, Samsung Medical Center, Sungkyunkwan University School of Medicine, Seoul, Republic of Korea. ${ }^{3}$ Department of Laboratory Medicine, Seoul National University College of Medicine, Seoul National University Bundang Hospital, Seongnam, Republic of Korea.

\section{Received: 29 August 2015 Accepted: 14 April 2016} Published online: 21 April 2016

\section{References}

1. Brushia RJ, Walsh DA. Phosphorylase kinase: the complexity of its regulation is reflected in the complexity of its structure. Front Biosci. 1999:4:D618-41.

2. Carriere C, Jonic S, Mornon JP, Callebaut I. 3D mapping of glycogenosiscausing mutations in the large regulatory alpha subunit of phosphorylase kinase. Biochim Biophys Acta. 2008;1782(11):664-70.

3. Roscher A, Patel J, Hewson S, Nagy L, Feigenbaum A, Kronick J, Raiman J, Schulze A, Siriwardena K, Mercimek-Mahmutoglu S. The natural history of glycogen storage disease types VI and IX: Long-term outcome from the largest metabolic center in Canada. Mol Genet Metab. 2014;113(3):171-6.

4. Tsilianidis LA, Fiske LM, Siegel S, Lumpkin C, Hoyt K, Wasserstein M, Weinstein DA. Aggressive therapy improves cirrhosis in glycogen storage disease type IX. Mol Genet Metab. 2013;109(2):179-82.

5. Johnson AO, Goldstein JL, Bali D. Glycogen storage disease type IX: novel PHKA2 missense mutation and cirrhosis. J Pediatr Gastroenterol Nutr. 2012;55(1):90-2

6. Davit-Spraul A, Piraud M, Dobbelaere D, Valayannopoulos V, Labrune P, Habes D, Bernard O, Jacquemin E, Baussan C. Liver glycogen storage diseases due to phosphorylase system deficiencies: diagnosis thanks to non invasive blood enzymatic and molecular studies. Mol Genet Metab. 2011:104(1-2):137-43.

7. Kishnani PS, Austin SL, Abdenur JE, Arn P, Bali DS, Boney A, Chung WK, Dagli Al, Dale D, Koeberl D, et al. Diagnosis and management of glycogen storage disease type I: a practice guideline of the American College of Medical Genetics and Genomics. Genet Med. 2014;16(11), e1.

8. HendrickX J, Lee P, Keating JP, Carton D, Sardharwalla IB, Tuchman M, Baussan C, Willems PJ. Complete genomic structure and mutational spectrum of PHKA2 in patients with $x$-linked liver glycogenosis type I and II. Am J Hum Genet. 1999;64(6):1541-9.

9. Pagon RA, Adam MP, Ardinger HH, Bird TD, Dolan CR, Fong C-T, Smith RJ, Stephens K, Goldstein J, Austin S. Phosphorylase Kinase Deficiency. In: GeneReviews. 2011. http://www.ncbi.n/m.nih.gov/books/NBK55061/. Accessed 27 Nov 2015

10. Song MJ, Lee ST, Lee MK, Ji Y, Kim JW, Ki CS. Estimation of carrier frequencies of six autosomal-recessive Mendelian disorders in the Korean population. J Hum Genet. 2012;57(2):139-44.

11. Jeong YJ, Kang B, Choi SY, Ki CS, Lee SY, Park HD, Choe YH. Does type I truly dominate hepatic glycogen storage diseases in Korea?: a single center study. Pediatr Gastroenterol Hepatol Nutr. 2014;17(4):239-47.

12. Shin YS. Diagnosis of glycogen storage disease. J Inherit Metab Dis. 1990:13(4):419-34

13. Park KJ, Park HD, Lee SY, Ki CS, Choe YH. A novel PHKA2 gross deletion mutation in a Korean patient with X-linked liver glycogenosis type I. Ann Clin Lab Sci. 2011;41(2):197-200

14. Kim JW, Kim JQ, Cho HI, Kim SI. Experience on the confirmatory enzyme tests for the Diagnosis of Glycogen Storage Disease. Korean J Clin Pathol. 1990;10(2):257-64

15. Desmet FO, Hamroun D, Lalande M, Collod-Beroud G, Claustres M, Beroud C. Human Splicing Finder: an online bioinformatics tool to predict splicing signals. Nucleic Acids Res. 2009;37(9):e67.

16. Korea Centers for Disease Control and Prevention, The Korean Pediatric Society, The Committee for the Growth Standard for Korean Children and Adolescents: 2007 Korean Children and Adolescents Growth Standard; 2007.

17. Thornton PS, Stanley CA, De Leon DD, Harris D, Haymond MW, Hussain K, Levitsky LL, Murad MH, Rozance PJ, Simmons RA, et al. Recommendations from the Pediatric Endocrine Society for Evaluation and Management of Persistent Hypoglycemia in Neonates, Infants, and Children. J Pediatr. 2015;167(2):238-45

18. Beauchamp NJ, Dalton A, Ramaswami U, Niinikoski H, Mention K, Kenny P, Kolho KL, Raiman J, Walter J, Treacy E, et al. Glycogen storage disease type IX: High variability in clinical phenotype. Mol Genet Metab. 2007;92(1-2):88-99.

19. Kim JA, Kim JH, Lee BH, Kim GH, Shin YS, Yoo HW, Kim KM. Clinical, Biochemical, and Genetic Characterization of Glycogen Storage Type IX in a Child with Asymptomatic Hepatomegaly. Pediatr Gastroenterol Hepatol Nutr. 2015;18(2):138-43.

20. Burwinkel B, Hu B, Schroers A, Clemens PR, Moses SW, Shin YS, Pongratz D, Vorgerd M, Kilimann MW. Muscle glycogenosis with low phosphorylase kinase activity: mutations in PHKA1, PHKG1 or six other candidate genes explain only a minority of cases. Eur J Hum Genet. 2003:11(7):516-26.

21. Goldstein J, Austin S, Kishnani P, Bali D. Phosphorylase Kinase Deficiency. In: Pagon RA, Adam MP, Ardinger HH, Wallace SE, Amemiya A, Bean LJH, Bird TD, Dolan CR, Fong CT, Smith RJH, et al., editors. GeneReviews. Seattle: University of Washington; 1993.

22. Achouitar S, Goldstein JL, Mohamed M, Austin S, Boyette K, Blanpain FM, Rehder CW, Kishnani PS, Wortmann SB, den Heijer M, et al. Common mutation in the PHKA2 gene with variable phenotype in patients with liver phosphorylase b kinase deficiency. Mol Genet Metab. 2011;104(4):691-4.

23. Brown LM, Corrado MM, van der Ende RM, Derks TG, Chen MA, Siegel S, Hoyt K, Correia CE, Lumpkin C, Flanagan TB, et al. Evaluation of glycogen storage disease as a cause of ketotic hypoglycemia in children. J Inherit Metab Dis. 2015;38(3):489-93.

24. Cho SY, Lam CW, Tong SF, Siu WK. X-linked glycogen storage disease IXa manifested in a female carrier due to skewed $X$ chromosome inactivation. Clin Chim Acta. 2013;426:75-8.

25. Ban K, Sugiyama K, Goto K, Mizutani F, Togari H. Detection of PHKA2 gene mutation in four Japanese patients with hepatic phosphorylase kinase deficiency. Tohoku J Exp Med. 2003;200(1):47-53.

26. Tuchman M, Brown BI, Burke BA, Ulstrom RA. Clinical and laboratory observations in a child with hepatic phosphorylase kinase deficiency. Metabolism. 1986;35(7):627-33.

27. Xiong HY, Alipanahi $B$, Lee $\sqcup$, Bretschneider $H$, Merico D, Yuen RK, Hua Y, Gueroussov S, Najafabadi HS, Hughes TR, et al. RNA splicing. The human splicing code reveals new insights into the genetic determinants of disease. Science. 2015;347(6218):1254806

28. Goldstein J, Austin S, Kishnani P, et al. Phosphorylase Kinase Deficiency. In: Pagon RA, Adam MP, Ardinger HH, et al., editors. GeneReviews ${ }^{\oplus}$. Seattle: University of Washington; 2011

29. Fukao $T$, Zhang G, Aoki Y, Arai T, Teramoto T, Kaneko H, Sugie H, Kondo N Identification of Alu-mediated, large deletion-spanning introns 19-26 in PHKA2 in a patient with X-linked liver glycogenosis (hepatic phosphorylase kinase deficiency). Mol Genet Metab. 2007;92(1-2):179-82.

30. Tarpey PS, Smith R, Pleasance E, Whibley A, Edkins S, Hardy C, O'Meara S, Latimer C, Dicks E, Menzies A, et al. A systematic, large-scale resequencing screen of $\mathrm{X}$-chromosome coding exons in mental retardation. Nat Genet. 2009;41(5):535-43.

31. Lau CK, Hui J, Fong FN, To KF, Fok TF, Tang NL, Tsui SK. Novel mutations in PHKA2 gene in glycogen storage disease type IX patients from Hong Kong China. Mol Genet Metab. 2011;102(2):222-5

32. Hirono H, Shoji Y, Takahashi T, Sato W, Takeda E, Nishijo T, Kuroda Y, Nishigaki T, Inui K, Takada G. Mutational analyses in four Japanese families with X-linked liver phosphorylase kinase deficiency type 1. J Inherit Metab Dis. 1998;21(8):846-52

33. Hidaka $\mathrm{F}$, Sawada $\mathrm{H}$, Matsuyama M, Nunoi $\mathrm{H}$. A novel mutation of the PHKA2 gene in a patient with X-linked liver glycogenosis type 1. Pediatr Int. 2005:47(6):687-90

34. van den Berg IE, van Beurden EA, Malingre HE, van Amstel HK, Poll-The BT, Smeitink JA, Lamers WH, Berger R. X-linked liver phosphorylase kinase deficiency is associated with mutations in the human liver phosphorylase kinase alpha subunit. Am J Hum Genet. 1995:56(2):381-7. 